\title{
PREMIERS ÉLÉMENTS DE CARACTÉRISATION GÉNÉTIQUE DE L'ALOSE DU RHÔNE (ALOSA FALLAX RHODANENSIS ROULE, 1924).
}

\author{
M. LE CORRE (1-2), D. LINHARES (3-4), F. CASTRO (3-4), P. ALEXANDRINO (3-4), \\ R. SABATIÉ (1-2), J.L. BAGLINIERE (1)
}

(1) INRA, Laboratoire d'Ecologie Aquatique, 65 rue de Saint-Brieuc, 35042 Rennes Cedex, France.

(2) ENSAR, Laboratoire d'Halieutique, 65 rue de Saint-Brieuc, 35042 Rennes Cedex, France.

(3) CECA, ICETA, Université de Porto, Campus Agrario de Vairao, R. Monte-Crasto, 4480 Vila do Conde, Portugal.

(4) Département de Zoologie et Anthropologie, Faculté des Sciences, Université de Porto, Pr. Gomez Teixeira, 4000 Porto, Portugal.

\section{RÉSUMÉ}

La systématique des deux espèces du genre Alosa ( $A$. alosa Linné, 1758 -grande alose- et $A$. fallax Lacépède, 1803 -alose feinte-) colonisant l'Atlantique-est et la Méditerranée reste encore confuse en l'absence de caractérisation génétique. En particulier, six sous-espèces ont été reconnues chez l'alose feinte uniquement à partir de critères morphométriques et méristiques. Parmi ces sous-espèces, l'une d'entre elles (Alosa fallax rhodanensis Roule, 1924 -alose du Rhône-) est inféodée au bassin méditerranéen français et notamment au fleuve Rhône. Les critères morphométriques et méristiques de ce taxon le rattachent sans équivoque à $A$. fallax. Cependant, son gabarit moyen et certaines de ses caractéristiques écologiques le rapprochent fortement d'A. alosa. Ainsi, pour connaître la véritable identité de l'alose du Rhône, une première caractérisation génétique a été entreprise à l'aide d'une analyse électrophorétique des protéines du foie (gel d'amidon) et du sang (isoélectrofocalisation) prélevées sur des échantillons d'adultes.

Dans un premier temps, deux des loci les plus performants pour différencier les deux aloses atlantiques (MPI, Mannose Phosphate Isomérase et HBA, chaîne $\alpha$ de l'Hémoglobine) ont été utilisés. L'observation des fréquences allèliques montre que l'alose du Rhône semble appartenir à l'espèce $A$. fallax. Dans un deuxième temps, un troisième locus (ADH, Alcool Déshydrogénase) a été utilisé car il a été reconnu comme permettant de discriminer les populations d'alose feinte du Portugal. L'expression de sa fréquence allèlique montre que la population du Rhône resterait proche de celle des fleuves du nord du Portugal.

Ces premiers résultats sont discutés en fonction des récentes études génétiques réalisées sur les aloses de l'Atlantique. Elles montrent que, compte tenu du caractère particulier de ce taxon, sa caractérisation génétique doit être complétée et approfondie.

Mots-clés : alose feinte, polymorphisme enzymatique, adulte, fleuve, Rhône. 


\section{PRELIMINARY DATA ON THE GENETIC CHARACTERIZATION OF TWAITE SHAD (ALOSA FALLAX RHODANENSIS ROULE, 1924) POPULATION IN THE RIVER RHONE.}

\section{ABSTRACT}

Two species of the genus Alosa ( $A$. alosa Linné, 1758 -allis shad- and $A$. fallax Lacépède, 1803 -twaite shad-) colonized the East Atlantic and the Mediterranean Sea. Their systematics is still confused without genetic characterization. Indeed, six subspecies of $A$. fallax are actually identified only on the basis of morphometric and meristic characters. One of these subspecies ( $A$. fallax rhodanensis Roule, 1924) is endemic of the Rhone River. Although this taxon has the same morphometric and meristic characters as A. fallax, it is closer to A. alosa for the size and the ecological features. So a genetic study, with electrophoretic analysis of liver (electrophoresis in starch gel) and blood (isoelectric focusing in polyacrylamid gel) proteins, has been carried out to elucidate the taxonomic status of this population.

We used two of the most discriminating loci between the two Atlantic shad species: MPI (Mannose Phospho Isomerase) and HBA ( $\alpha$ haemoglobin chain). Allelic frequency puts in conspicuous the appartenance of $A$. fallax rhodanensis to the taxon $A$. fallax. Then, we used a locus known to discriminate the populations of $A$. fallax in Atlantic (ADH : Alcohol Deshydrogenase). Through $A D H$ allelic frequency, twaite shad from the Rhone River is close to populations of the north of Portugal.

Results are discussed according to recent genetic studies made on Atlantic shads. They showed that further information had to be regarded on the genetic characteristics of this allis shad in relation to its particular status.

Key-words : twaite shad, enzyme polymorphism, adult, river, Rhone River.

\section{INTRODUCTION}

La systématique des deux espèces du genre Alosa ( $A$. alosa, la grande alose et A. fallax, l'alose feinte) colonisant l'Atlantique-est et la Méditerranée demeure assez confuse. En particulier, chez les aloses feintes, six sous-espèces ont été reconnues jusqu'à présent uniquement à partir de critères morphométriques et méristiques (QUIGNARD et KARTAS, 1977). Parmi tous ces critères, le nombre de branchiospines sur le premier arc branchial reste le plus performant pour l'identification (MENNESSONBOISNEAU et BOISNEAU, 1990 ; TAVERNY, 1991 ; SABATIE, 1993 ; ALEXANDRINO, 1996). Ainsi, la grande alose possède en moyenne 90 à 155 branchiospines (QUIGNARD et DOUCHEMENT, 1991a), les aloses feintes de l'Atlantique 40 à 50 (QUIGNARD et DOUCHEMENT, 1991b) et pour celles de la Méditerranée, cette valeur peut varier de 28 à 60 en fonction des sous-espèces considérées (KARTAS, 1991).

Parmi ces sous-espèces, il en existe une inféodée à la partie nord-est et occidentale de la Méditerranée, l'alose feinte du Rhône (Alosa fallax rhodanensis, Roule 1924) (QUIGNARD et KARTAS, 1977). Ce taxon a toujours posé un certain nombre de difficultés quant à sa position systématique. En effet, ROULE (1924) le considérait comme une espèce à part entière, tandis que QUIGNARD et KARTAS (1977) l'ont identifié comme une sous-espèce d'A. fallax. Actuellement, c'est cette nomenclature qui est le plus souvent reprise (QUIGNARD et DOUCHEMENT, 1991c). Pourtant, WHITEHEAD (1985), dans sa classification FAO du genre Alosa, ne reconnaît que deux sous-espèces : A. fallax fallax présente dans l'Atlantique et $A$. fallax nilotica colonisant le pourtour du bassin 
méditerranéen. De même plus récemment, BIANCO (1995) émettait des réserves quant à l'identification de l'alose du Rhône comme sous-espèce méditerranéenne. Ces changements et ces incertitudes quant à la position de ce taxon proviennent, en fait, de ses caractéristiques biologiques. En effet, les critères méristiques le rattachent sans ambiguïté à l'espèce $A$. fallax alors que ces paramètres biologiques (grande taille, longue distance de migration en eau douce avant la mise en place de barrages (HOESTLANDT, 1958)) le rapprochent très fortement d'A. alosa (LE CORRE et al., 1997).

A cette position taxonomique confuse s'ajoute l'incertitude quant à la présence de grandes aloses sur le bassin du Rhône. La majorité des auteurs s'accordait à considérer sa présence comme très douteuse (ROULE, 1924 ; GALLOIS, 1946 ; HOESTLANDT, 1958). Mais HOESTLANDT (1958) a effectué en 1948 un transfert d'A. alosa de la Dordogne vers le Rhône. Ce transfert d'individus de grandes aloses, que l'auteur considère comme réussi bien qu'aucun suivi n'ait été effectué, a pu modifier la situation sur le Rhône. Ainsi DOUCHEMENT (1981) a identifié, à partir de critères méristiques, trois groupes taxonomiques sur le Rhône : la grande alose, bien que leur nombre de branchiospines soit nettement inférieur à ce qui est habituellement décrit pour cette espèce (en moyenne 70 ), l'alose du Rhône ( $A$. fallax rhodanensis) et son hybride avec $A$. alosa présentant un nombre de branchiospines intermédiaire entre celui compté pour chacune de ces deux espèces.

Afin de clarifier la situation taxonomique de l'alose du Rhône, nous avons entrepris une première caractérisation génétique à partir d'électrophorèse de protéines du foie et du sang. Cette étude s'inscrit dans un programme plus large qui a débuté en 1995 ayant pour but d'améliorer l'état des connaissances écologiques et biologiques de ce taxon. Cette première étude génétique prend en compte trois loci : celui de la Mannose Phosphate Isomérase (MPI) et celui de la chaîne $\alpha$ de l'Hémoglobine (HBA) qui permettent de discriminer les deux espèces du genre Alosa, puis celui de l'Alcool Déshydrogénase $(A D H)$ qui permet de séparer les populations d'alose feinte (ALEXANDRINO, 1996). Ces premiers éléments de caractérisation génétique sont ensuite comparés à ceux obtenus sur les populations de grandes aloses et d'aloses feintes de quelques fleuves atlantiques (ALEXANDRINO, 1996).

\section{MATÉRIEL ET MÉTHODES}

\section{Récolte des échantillons}

Un échantillon d'aloses du Rhône a été récolté par pêche au filet à l'aval immédiat du barrage de Vallabrègues situé à environ $60 \mathrm{~km}$ de la mer (Bouches-du-Rhône, France). Les pêches se sont déroulées sur toute la période de migration, entre le mois de mars et le mois de mai 1997 et 45 individus (dont 22 mâles et 23 femelles) ont été capturés.

Chaque individu a été sexé, mesuré, pesé, et a fait l'objet de comptages méristiques en particulier le nombre de branchiospines sur le premier arc branchial gauche.

Sur tous les individus capturés, des échantillons de sang et de foie ont été prélevés. Pour le sang, la séparation entre érythrocytes et plasma a été réalisée immédiatement après prélèvement par centrifugation ( $3000 \mathrm{tr} / \mathrm{min}$ pendant 15 minutes). Ces échantillons ont été conservés à $-80^{\circ} \mathrm{C}$ avant d'être analysés.

\section{Techniques électrophorétiques}

- MPI (Mannose Phosphate Isomérase) et ADH (Alcool Déshydrogénase) : I'analyse de ces loci a été effectuée à partir d'échantillon de foie à l'aide d'électrophorèses sur gel d'amidon selon la méthode décrite par ALEXANDRINO (1996). 
- HBA (chaîne a de l'Hémoglobine) : l'analyse de ce locus a été faite sur les érythrocytes par une technique d'isoélectrofocalisation sur gel de polyacrilamide selon la méthode décrite par ALEXANDRINO (1996).

\section{Traitement des données}

La distribution du nombre de branchiospines a été étudiée afin de repérer, le cas échéant, plusieurs groupes d'individus dans la population échantillonnée (logiciel SYSTAT).

Les trois loci étudiés sont diallèliques. Ainsi, le locus MPI peut être sous la forme $M \mathrm{MPI}^{\star} 1$ ou MPI${ }^{\star} 2$, le locus HBA sous celle HBA*S ou HBA* $\mathrm{F}$ et le locus $\mathrm{ADH}$ sous celle $\mathrm{ADH}^{\star} 1$ ou $\mathrm{ADH}^{\star} 2$.

Les fréquences allèliques, les valeurs des taux d'hétérozygotie des populations et les distances génétiques ont été calculées suivant les critères de NEl (1973 et 1975) avec le logiciel BIOSYS.

La diversité génétique totale $\left(\mathrm{H}^{\top}\right)$ a été décomposée en trois fractions :

- $\mathrm{G}_{p}$ : part de la diversité imputable à la variation entre individus au sein des populations,

- $\mathrm{G}_{\mathrm{PS}}$ : part de la diversité imputable à la variation entre les populations au sein des espèces,

- $\mathrm{G}_{\mathrm{ST}}$ : part de la diversité imputable à la variation entre les espèces au sein du groupe étudié.

Les résultats obtenus sur l'échantillon rhodanien ont été comparés avec ceux obtenus pour les populations d'A. alosa de la Garonne, du Lima et du Mondego et d'A. fallax du Lima, du Mondego, du Tage, du Mira et du Guadiana (ALEXANDRINO, 1996).

\section{RÉSULTATS}

\section{Distribution du nombre de branchiospines}

La distribution du nombre de branchiospines ne fait apparaître qu'un seul groupe (Figure 1) dont le nombre moyen de branchiospines est 37,96 (écart type : 1,66).

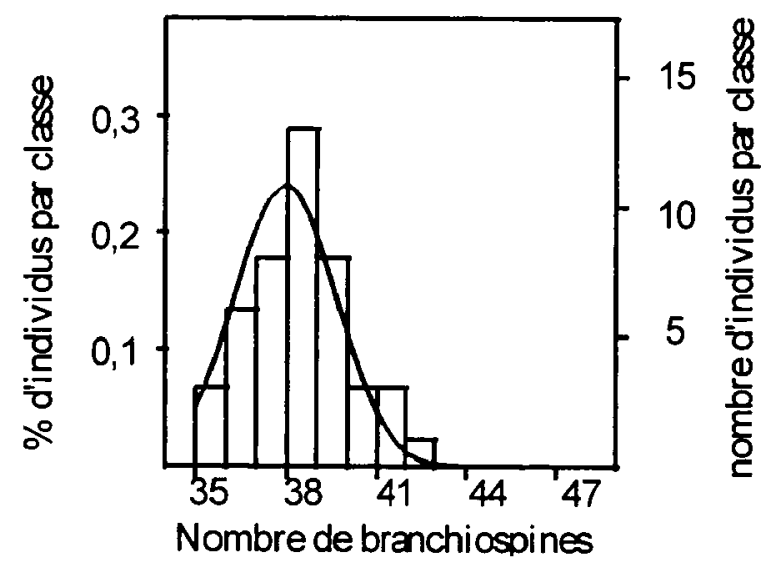

Figure 1

Distribution du nombre de branchiospines des aloses échantillonnées sur le Rhône.

\section{Figure 1}

Distribution of individual gillraker number in shad samples from the Rhone River. 


\section{Fréquence phénotypique de la population d'alose du Rhône}

L'analyse des fréquences phénotypiques de l'échantillon rhodanien montre que les loci MPI et ADH sont monomorphes, tous les individus sont donc homozygotes pour ces deux loci (Tableau I).

Un individu hétérozygote (FS) est mis en évidence par l'analyse du locus HBA pour lequel les valeurs observées correspondent aux valeurs théoriques. Cependant, l'effectif de la classe représentant l'allèle rare est trop faible pour que l'écart à l'équilibre de Hardy-Weinberg soit testé (Tableau I).

La population du Rhône a donc un pourcentage de loci polymorphes de 33,3\% au critère 0,99 (c'est-à-dire que la fréquence de l'allèle le plus commun n'excède pas 0,99 ) et de $0 \%$ au critère 0,95 .

\section{Tableau I}

Effectif des différents phénotypes observés (n obs.) et calculés ( $n$ th.) rencontrés dans l'échantillon d'aloses capturées sur le Rhône.

\section{Table I}

Observed ( $n$ obs.) and expected ( $n$ th.) phenotypic distributions in shad sample caught in the Rhone River.

\begin{tabular}{c|ccc|ccc|ccc}
\hline Loci & \multicolumn{3}{c}{ MPI } & \multicolumn{4}{c}{ HBA } & ADH \\
phénotype & 11 & 12 & 22 & SS & SF & FF & 11 & 12 & 22 \\
n obs. & 42 & 0 & 0 & 44 & 1 & 0 & 40 & 0 & 0 \\
n th. & & & & & 44 & 1 & 0 & & \\
\hline
\end{tabular}

\section{Fréquence allèlique}

Les fréquences allèliques calculées sur les loci MPI et HBA pour les différentes populations d'alose feinte et de grande alose montrent que la population d'alose du Rhône est génétiquement plus proche d'A. fallax que d'A. alosa. En effet, pour le locus MPI, l'allèle le plus fréquent chez $A$. fallax: MPI*1 (fréquence variant de 0,646 à 0,857 en fonction des populations) est fixé chez la population rhodanienne alors qu'il est très rare (fréquence $=0,098$ ) ou absent chez $A$. alosa (Figure 2a).

De la même manière, l'allèle HBA*S est largement majoritaire chez la population rhodanienne $(0,989)$ et les populations d'alose feinte du Portugal (fréquence variant de 0,850 à 1,000 ) alors qu'il est rare chez les populations de grande alose (fréquence variant de 0,000 à 0,150 ) (Figure $2 b$ ).

En ce qui concerne le locus $A D H$, l'allèle $A D H^{\star} 1$ est fixé pour les trois populations de grande alose prises en compte, pour la population rhodanienne et pour la population d'alose feinte du Lima. Dans les autres populations d'alose feinte, ce locus est polymorphe et la fréquence de l'allèle $\mathrm{ADH}^{*} 1$ varie de 0,205 à 0,706 (Figure 2c). 

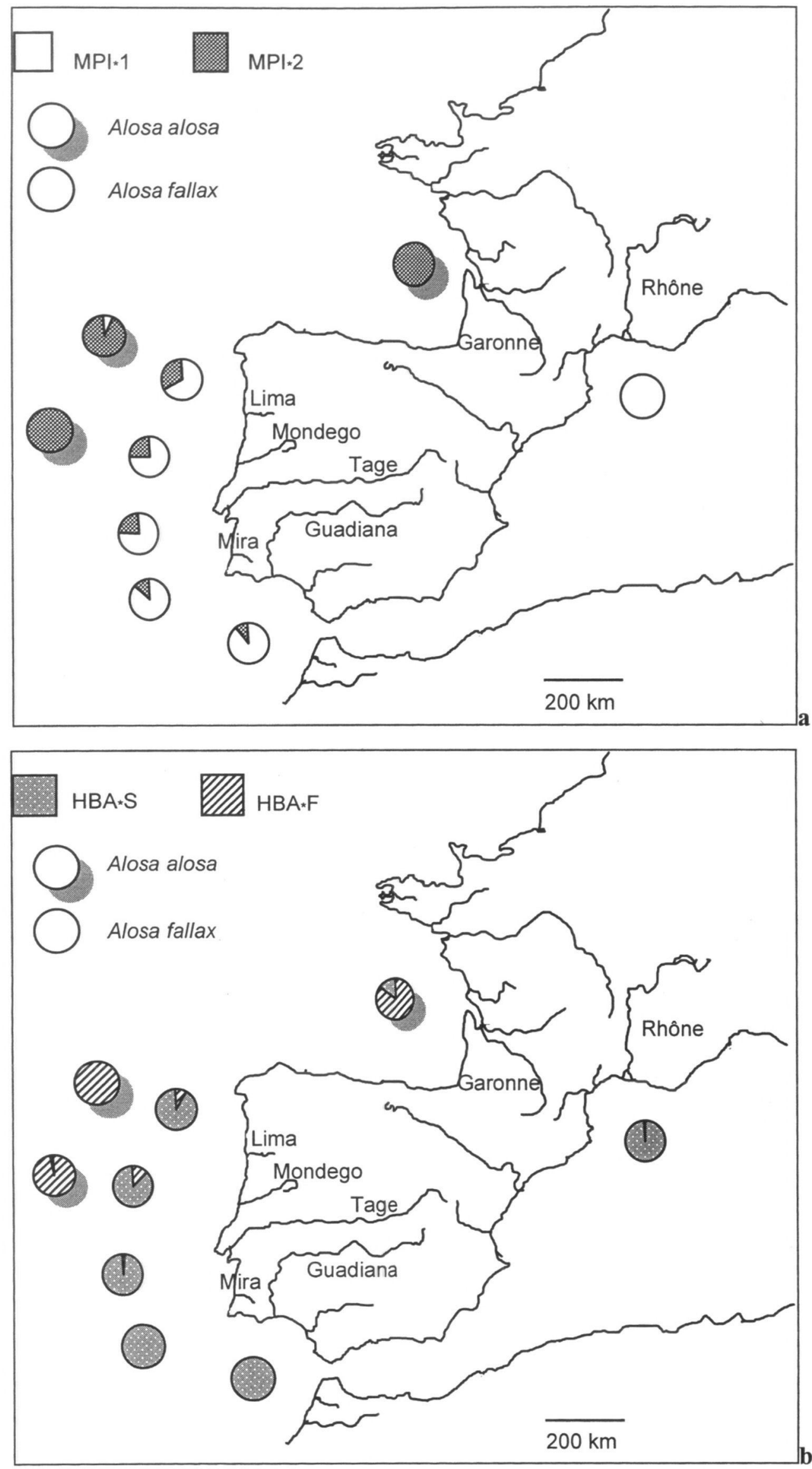


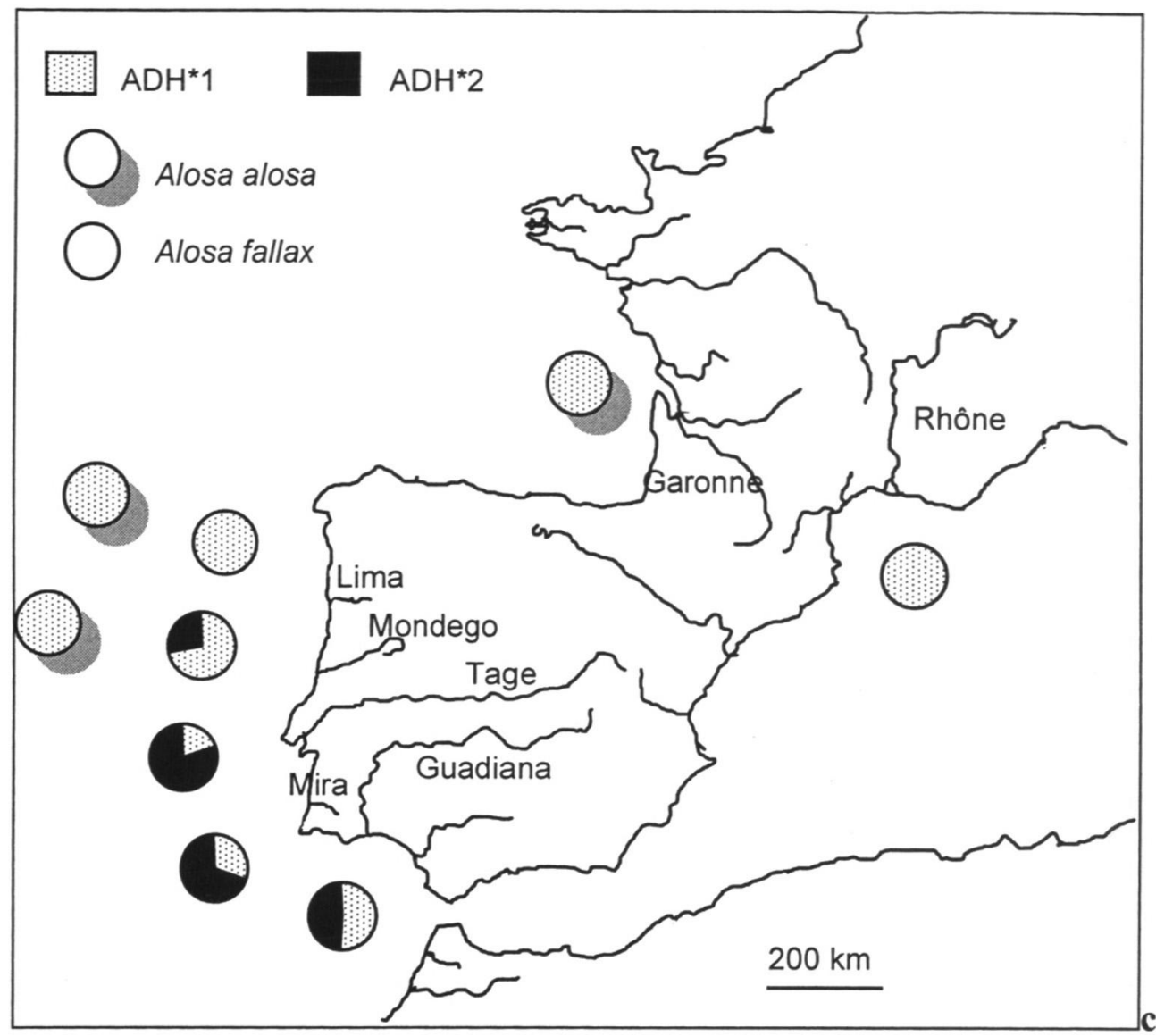

\section{Figure 2}

Fréquences allèliques des loci MPI (2a), HBA (2b) et ADH (2c) pour toutes les populations d'alose prises en compte.

\section{Figure 2}

Allelic frequencies of MPI (2a), HBA (2b) and ADH (2c) loci in different shad populations taken into account.

\section{La diversité génétique}

Deux groupes de loci se distinguent. Le premier est formé par HBA et MPI, où la plus grande partie de la diversité génétique provient de la différence entre les espèces $\left(G_{S T}\right.$ variant de $56,3 \%$ à $78,2 \%$ ). Le second est représenté uniquement par le locus $A D H$, dans ce cas la part de diversité due à la différence entre espèces est faible $\left(G_{\text {Sт }}\right.$ de $25,4 \%$ ) alors que la fraction de la variation due aux différences entre populations ( $G_{p S}$ de $25,7 \%$ ) est de l'ordre de 10 fois supérieure à ce qu'elle représente pour les autres loci $\left(G_{p s}\right.$ variant de 1,7 à $2,5 \%$ ) (Tableau II). 


\section{Tableau II}

Diversité génétique absolue $\left(\mathrm{H}_{T}\right)$ et relative $(\mathrm{G})$ (NEI, 1975) des populations d'alose étudiées.

\section{Table II}

Absolute $\left(\mathrm{H}_{\mathrm{T}}\right)$ and relative $(\mathrm{G})$ genetic diversity (NEI, 1975) for the studied shad populations.

\begin{tabular}{|c|c|c|c|c|}
\hline & \multirow[b]{2}{*}{$\begin{array}{c}\text { Diversité } \\
\text { génétique } \\
\text { totale } \\
\left(\mathrm{H}_{\mathrm{T}}\right)\end{array}$} & \multicolumn{3}{|c|}{ Diversité rolative (\%) } \\
\hline & & $\begin{array}{c}\text { intrapopulation } \\
\left(G_{p}\right)\end{array}$ & $\begin{array}{c}\text { interpopulation et } \\
\text { intraspécifique } \\
\left(G_{\mathrm{ps}}\right)\end{array}$ & $\begin{array}{c}\text { interspécifique } \\
\left(G_{\mathrm{s}}\right)\end{array}$ \\
\hline $\begin{array}{l}\text { MPI } \\
\text { HBA }\end{array}$ & $\begin{array}{l}0,495 \\
0,452\end{array}$ & $\begin{array}{l}41,2 \% \\
20,1 \%\end{array}$ & $\begin{array}{l}2,5 \% \\
1,7 \%\end{array}$ & $\begin{array}{l}56,3 \% \\
78,2 \%\end{array}$ \\
\hline $\mathrm{ADH}$ & 0,378 & $48,9 \%$ & $25,7 \%$ & $25,4 \%$ \\
\hline moyenne (3 loci) & 0,442 & $36,7 \%$ & $10 \%$ & $53,3 \%$ \\
\hline
\end{tabular}

\section{Distance génétique}

Les distances génétiques basées sur ces trois loci montrent que la population d'alose du Rhône est plus proche des aloses feintes que des grandes aloses. Ainsi, la distance génétique moyenne entre les grandes aloses et la population rhodanienne est de 0,974 , alors qu'elle n'est que de 0,138 entre cette population et les autres populations d'alose feinte (Tableau III).

De plus, les aloses du Rhône sont les plus proches des populations d'aloses feintes du nord du Portugal en particulier du Lima (distance génétique de 0,044) et dans une moindre mesure du Mondego (distance génétique de 0,051 ) que des autres populations (Tableau III).

\section{Tableau III}

Distances génétiques (NEI, 1972) entre les différentes populations d'alose étudiées (a. : A. alosa, f. : A. fallax, Garon. : Garonne, Lima : Lima, Mond. : Mondego, Rhône : Rhône, Tage : Tage, Guad. : Guadiana).

Table III

Genetic distances (NEI, 1972) between different shad populations (a. : A. alosa, f. : A. fallax, Garon. : Garonne River, Lima : Lima River, Mond. : Mondego River, Rhône : Rhone River, Tage : Tage River, Guad. : Guadiana River).

\begin{tabular}{|c|c|c|c|c|c|c|c|c|c|}
\hline & a. Garon. & a. Lima & a. Mond. & f. Rhờne & f. Lima & f. Mond. & f. Tage & f. Mira & f. Guad. \\
\hline a. Garon. & - & & & & & & & & \\
\hline a. Lima & 0,011 & -.............. & & & & & & & \\
\hline a. Mond. & 0,005 & 0,003 & - & & & & & & \\
\hline f. Rhơne & 0,904 & 0,972 & 1,046 & ............. & & & & & \\
\hline f. Lima & 0,483 & 0,562 & 0,582 & 0,044 & --.---.--. & & & & \\
\hline f. Mond. & 0,636 & 0,705 & 0,742 & 0,051 & 0,042 & -............ & & & \\
\hline f. Tage & 1,375 & 1,561 & 1,610 & 0,301 & 0,330 & 0,134 & $\cdots$ & & \\
\hline f. Mira & 1,429 & 1,599 & 1,186 & 0,198 & 0,254 & 0,090 & 0,011 & -.......... & \\
\hline f. Guad. & 1,188 & 1,317 & 1,394 & 0,097 & 0,146 & 0,038 & 0,048 & 0,016 & $\cdots$ \\
\hline
\end{tabular}




\section{DISCUSSION - CONCLUSION}

La distribution du nombre de branchiospines et sa valeur moyenne obtenues à partir de l'échantillon d'alose du Rhône ne font apparaître qu'un seul groupe d'individus que l'on peut rattacher à l'espèce $A$. fallax. Ces résultats sont en accord avec les observations faites à la suite de l'étude morphométrique et méristique de près de 2000 aloses du Rhône (LE CORRE et al., 1997). De plus l'analyse des fréquences allèliques pour les loci MPI et HBA montre clairement que les aloses du Rhône appartiennent au groupe des A. fallax.

Cependant, la présence d'un individu hétérozygote (FS) pour le locus HBA conduit à émettre deux hypothèses. En effet, chez les $A$. fallax, le locus HBA peut être diallèlique avec un allèle rare $(F)$ que l'on rencontre à une fréquence très faible chez de nombreuses populations (Lima, Mondego, Tage et Rhône) ou à une fréquence nulle chez les populations dont l'histoire (effet fondateur ou "bottle neck " par exemple) a entraîné la fixation de l'allèle le plus commun (Mira et Guadiana). Mais il est également possible qu'à l'origine, le locus HBA ait été monomorphe (S) chez $A$. fallax rhodanensis. L'introduction d'A. alosa (F fixé) par HOESTLANDT (1958) aurait alors permis l'apparition d'hybrides fertiles pouvant avoir des conséquences néfastes sur la préservation du patrimoine génétique originel. L'existence d'hybrides entre les deux espèces du genre Alosa est bien connue et se retrouve dans de nombreux bassins où elles sont en sympatrie (MENNESSON-BOISNEAU et BOISNEAU, 1990 ; ALEXANDRINO, 1996 ; ALEXANDRINO et al., 1996 ; SABATIE, 1993). Ces hybrides de première génération ( $F 1$ ), en se reproduisant avec des $A$. fallax rhodanensis, auraient permis à l'allèle $\mathrm{F}$ de se propager au sein des aloses du Rhône. Dans le cas où cette dernière hypothèse serait la bonne, la fréquence de l'allèle $F$ doit diminuer au cours du temps en l'absence de nouvel apport extérieur, à moins qu'il ne donne un avantage (meilleur " fitness ") aux individus qui en sont porteurs.

Les distances génétiques calculées font apparaître que les populations les plus proches des aloses du Rhône sont celles du nord du Portugal (Lima et Mondego). Ce rapprochement provient certainement des fréquences allèliques proches du locus $A D H$ pour ces populations. Or, le locus $A D H$ qui est le plus approprié pour discriminer des populations (ALEXANDRINO, 1996) semble mettre en évidence l'existence d'un gradient Nord/Sud dans les fréquences allèliques des différentes populations prises en compte dans cette étude. En effet, pour les populations les plus au nord ( $A$. alosa de la Garonne, du Lima, et du Mondego et les $A$. fallax du Rhône et du Lima) l'allèle $A D H^{*} 1$ est fixé alors que les populations plus méridionales sont polymorphes avec une tendance à l'augmentation de la fréquence de l'allèle $\mathrm{ADH}^{\star} 2$ quand on va vers le sud. En fait, les fréquences des différents allèles de ce locus apparaîtraient plus comme le résultat de l'action de facteurs environnementaux que reliées à l'histoire phylogénétique des taxons.

L'existence d'un seul groupe d'individus ( $A$. fallax rhodanensis) identifié à partir de caractères méristiques et génétiques va à l'encontre de la situation rencontrée par DOUCHEMENT (1981) dans les années 1970. II est en effet probable que les grandes aloses de la Dordogne introduites en 1948 sur le Rhône (HOESTLANDT, 1958) ne se soient pas maintenues très longtemps. Même si la présence d'un individu hétérozygote pour le locus HBA ne permet pas d'écarter totalement l'hypothèse d'une hybridation ancienne entre des grandes aloses et les aloses feintes du Rhône.

Cette première étude sur trois loci nous a permis de mettre en évidence l'appartenance génétique d' $A$. fallax rhodanensis au taxon $A$. fallax. La poursuite de ce travail, et en particulier la prise en compte d'autres loci, est maintenant nécessaire afin de confirmer ce résultat et d'appréhender plus finement les variations entre les différentes 
populations d'alose feinte sur leur aire de répartition, ainsi que de déterminer si le rang de sous-espèce doit être attribué aux aloses du Rhône ou si ce n'est qu'une population d'Alosa fallax parmi d'autres.

L'étude génétique doit donc être étendue à d'autres populations. En particulier à celles des côtes anglaises, de l'ouest de la France (Charente, Bretagne, Normandie) et du Maroc pour cerner toute l'aire atlantique de répartition. Ainsi qu'aux populations des fleuves situés à l'ouest de la Méditerranée tels que l'Aude en France ou l'Ebre en Espagne et aux populations italiennes aussi bien fluviales que lacustres. En effet, pour ces populations, des données relatives aux fréquences allèliques de nombreux loci seraient indispensables pour améliorer l'état des connaissances génétiques de ces deux espèces et pour appréhender les évolutions entre les populations méditerranéennes et atlantiques. II faudrait également pouvoir étudier les conditions environnementales (la température par exemple) des différents fleuves où sont présentes des populations d'aloses car ces facteurs pourraient jouer un rôle sur les fréquences allèliques du locus ADH.

\section{BIBLIOGRAPHIE}

ALEXANDRINO P., 1996. Estudo de populaçoes de savel (Alosa alosa L.) e savelha (Alosa fallax, Lacépède). Analise da diferenciaçao interspecifica, subestructuraçao e hibridaçao. Thèse de Doctorat, Université de Porto, $180 \mathrm{p}$.

ALEXANDRINO P., FERRAND N., ROCHA J., 1996. Genetic polymorphism of a haemoglobin chain and adenosine deaminase in European shads : evidence for the existence of two distinct genetic entities with natural hybridization. Journal of Fish Biology, 48, 447-456.

BIANCO P.G., 1995. Mediterranean endemic freshwater fishes of Italy. Biol. Conserv., 72, 159-170.

DOUCHEMENT C., 1981. Les aloses des fleuves français, Alosa fallax Lacépède, 1803 et Alosa alosa Linné, 1758. Biométrie, Ecobiologie, Autonomie des populations. Thèse de 3è cycle, Université des Sciences et Techniques du Languedoc, Montpellier, $377 \mathrm{p}$.

GALLOIS C., 1946. L'alose du Rhône. Bull. Fr. Pêche Piscic., 141, 162-176.

HOESTLANDT H., 1958. Reproduction de l'alose atlantique (Alosa alosa Linné) et transfert au bassin méditerranéen. Verhandlungen, Internationale Vereineigung für theorische und angewandte Limnologie, 13, 736-742.

KARTAS, 1991. Alosa fallax algeriensis (Regan, 1916). In HOESTLANDT H. (ed.), The freshwater fishes of Europe, vol. 2, Clupeidae, Anguillidae., Aula-Verlag, Wiesbaden, $448 \mathrm{p}$.

LE CORRE M., BAGLINIERE J.L., SABATIE R., MENELLA J.Y., PONT D., 1997. Données récentes sur les caractéristiques morphométriques et biologiques de la population d'Alose feinte du Rhône (Alosa fallax rhodanensis Roule, 1924). Bull. Fr. Pêche Piscic., 346, 527-545.

MENNESSON-BOISNEAU C., BOISNEAU P., 1990. Recherches sur les aloses du bassin de la Loire : Migration, Répartition, Reproduction, Caractéristiques biologiques et taxonomiques des aloses (Alosa sp.). Thèse de Doctorat (document de synthèse), Université de Rennes et de Paris-Val-de-Marne, $143 \mathrm{p}$.

NEI M., 1972. Genetic distance between populations. Am. Nat., 106, 283-292.

NEI M., 1973. Analysis of genes diversity in subdivided populations. Proc. Nat. Acad. Sci. U.S.A., 70, 3321-3323.

NEI M., 1975. Molecular population genetics and evolution. North-Holland. 
QUIGNARD J.P., DOUCHEMENT C., 1991a. Alosa alosa (Linaeus, 1758). In HOESTLANDT $H$. (ed.), The freshwater fishes of Europe, vol. 2, Clupeidae, Anguillidae, Aula-Verlag, Wiesbaden, $448 \mathrm{p}$.

QUIGNARD J.P., DOUCHEMENT C., 1991b. Alosa fallax fallax (Lacépède, 1803). In HOESTLANDT $H$. (ed.), The freshwater fishes of Europe, vol. 2, Clupeidae, Anguillidae, Aula-Verlag, Wiesbaden, $448 \mathrm{p}$.

QUIGNARD J.P., DOUCHEMENT C., 1991c. Alosa fallax rhodanensis (Lacépède, 1803). In HOESTLANDT H. (ed.), The freshwater fishes of Europe, vol. 2, Clupeidae, Anguillidae, Aula-Verlag, Wiesbaden, $448 \mathrm{p}$.

QUIGNARD J.P., KARTAS F., 1977. Les aloses feintes, Alosa fallax (Lacépède, 1803), poissons clupéiformes de l'Atlantique nord-est et de la Méditerranée : Etude des caractères numériques. Bull. MNHN, $3^{\text {eme }}$ série, 501, 1241-1256.

ROULE L., 1924. Les aloses des eaux douces de France. Bull. Soc. Zool. France, 49, 265-266.

SABATIE M.R., 1993. Recherches sur la biologie et l'écologie des aloses au Maroc (Alosa alosa, Linné, 1758 et Alosa fallax Lacépède, 1803) : Exploitation et taxonomie des populations atlantiques, Bioécologie des aloses de l'Oued Sébou. Thèse de Doctorat, Université de Bretagne Occidentale, Brest, $326 \mathrm{p}$.

TAVERNY C., 1991. Contribution à la connaissance de la dynamique de populations d'aloses (Alosa alosa et Alosa fallax) dans le système fluvio-estuarien de la Gironde : Pêche, Biologie, Ecologie. Thèse Université de Bordeaux I, $451 \mathrm{p}$.

WHITEHEAD P.J.P., 1985. FAO species catalogue. 7 : Clupeoid fishes of the world (Suborder Clupeoidei). An annoted and illustred catalogue of the Herrings, Sardines, Pilchards, Sprats, Anchovies and Wolf-Herrings. Part 1 : Chirocentridae, Clupeidae and Pristigasteridae. FAO Fisheries Synopsis, 125, 7, Part 1, 303 p. 